\title{
New T-helper subsets controlling the immune system and tissue cells
}

\author{
"The discovery of more T-cell subsets may complicate our view on specific immune \\ responses and the fact that some of the mediators are overlapping raises the question of \\ whether the definition of new or any subset makes any sense."
}

The immune system has evolutionarily developed a specific recognition system that is characterized by long-lived cells carrying highly polymorphic receptors. These receptors either recognize native proteins (B cells) or fragments of proteins (T cells). Both of these long-lived cell types form the immunologic memory; this is a key target for immunotherapies to hopefully sustain treatment effects in T- or B-cell-dependent diseases such as allergies or autoimmune diseases. Originally, T cells were discovered to be essential for immunoglobulin induction and 'helping' $\mathrm{B}$ cells to differentiate IgM from IgG or IgE production. Two phenotypically different $T$-cell subsets were discovered, namely the IFN- $\gamma$-producing Th1 and the IL-4-secreting Th2 cells. A dichotomic Th1/Th2 paradigm has dominated our view on allergy pathogenesis and has produced important concepts such as the hygiene hypothesis. This dichotomic view has been dramatically changed with the discovery of regulatory T cells, Th17 [1] and Th22 cells.

\section{T-cell differentiation \& polarization}

An important note to understand any helper subset is that all T-helper cells are believed to originate from naive $\mathrm{T}$ cells, which differentiates upon initial antigen challenge [2]. Thus, interventions aiming at this fundamental process must take into consideration that any drug that aims to alter this process must coincide its action with the recognition of the specific T-cell peptide presented by an antigen-presenting cell [3-5]. Furthermore, this antigen contact needs to induce T-cell proliferation, which is a prerequisite of T-cell differentiation. The polarization involves a competitive and exclusive mechanism of differentiation that generally results in discrete phenotypes. The exclusiveness of this polarization mechanism has been studied in quite some detail for Th1 and
Th2 cells [6], but also appears to be valid for other T-cell subsets. It is known, for example, that Th2 differentiation inhibits Th1 commitment and vice versa. Specifically, the IL-4-induced transcription factors STAT-6 and GATA-3 inhibit differentiation into Th1 cells in the early phase of commitment $[7,8]$. Recently, it was shown that the Th1 transcription factor T-bet directly regulates GATA-3 function, suggesting that transcription factors compete in the early differentiation phase of $\mathrm{T}$ cells, potentially integrating environmental signals in order to finally imprint the T-cell phenotype $[9,10]$. The imprinted phenotype is thought to be based on epigenetic modification that provides a stable phenotype even upon multiple cell divisions, which explains how clonal expansion can be realized after phenotypic T-cell differentiation. Therefore, immunomodulation on the path of T-cell differentiation is likely to generate sustained treatment effects, but is always dependent on effective antigen stimulation or vaccination.

\section{Regulatory T cells}

The Tregs are defined by a functional ability to inhibit effector cells such as Th1, Th2 or Th17 cells. The impact on Th22 cells is still unclear, but is assumed to be similar to those of other subsets. To date, it is not fully clear whether there are different subsets of Tregs or whether Tregs are more variable in their expression of suppressive cytokines such as IL-10, TGF- $\beta$ or cytotoxic T-lymphocyte antigen 4. Owing the association of Tregs with immune tolerance, it is tempting to propose immunotherapies that induce Tregs and thus promote endogenous anti-inflammatory mechanisms in an antigen-specific fashion. In fact, allergen-specific immunotherapy has been shown to induce or expand Tregs [11-15]. Foxp3 is a critical transcription factor for the differentiation of Tregs, which has been reviewed elsewhere [16].

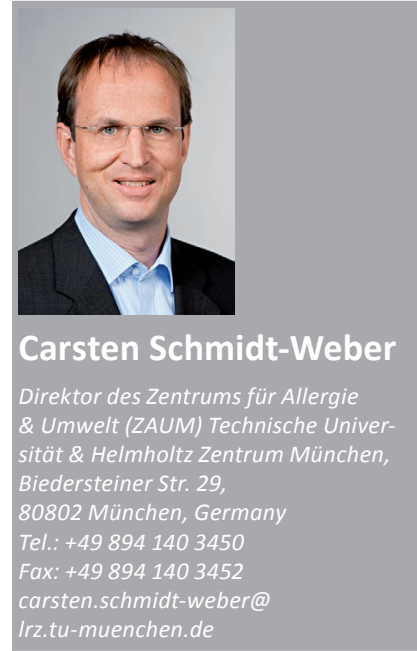

future medictio $^{\text {pisg }}$ 
It is effectively suppressed by the Th2 transcription factor GATA-3, suggesting that Th2-driven inflammation can prevent Treg-mediated tolerance induction. Since bare antigen stimulation of T cells generally induces Foxp3, we hypothesize that Treg induction may represent a default pathway that only deviates into proinflammatory effector (Th1, Th2 and Th17 cells) if proinflammatory cytokines are present during the initial differentiation process.

\section{Th17 cells}

The Th17 cell subset is characterized by IL-17A/F, IL-22 secretion and the expression of the transcription factor RORC2 (ROR $\gamma t$ in the murine context; for review see [17]). However, both cytokines are also expressed by other T-cell subsets. To distinguish Th17 from other IL-17-expressing subsets, the expression of the IL-23R is also an important determinant. The discovery of Th17 cells changed our understanding of Th1 cells, which were previously considered as the main drivers of nonallergic, acute inflammation. However, the discovery of Th17 cells revealed their key role in the tissue changes observed in major inflammatory diseases such as arthritis. In fact, recent data even suggest that by contrast to their previously suggest proinflammatory role, Th1 cytokines such as IFN- $\gamma$ and IL-27 are enhancers of Foxp3 and may also be important in initiating Treg responses, possibly by enhancing the MHC II-dependent antigen presentation [18].

By contrast to other T-helper subsets, Th17 cells mainly act on tissue cells according to the IL-17A/F receptor expression. IL-17 induces IL-8 in epithelial cells [19], which in turn recruits neutrophils to

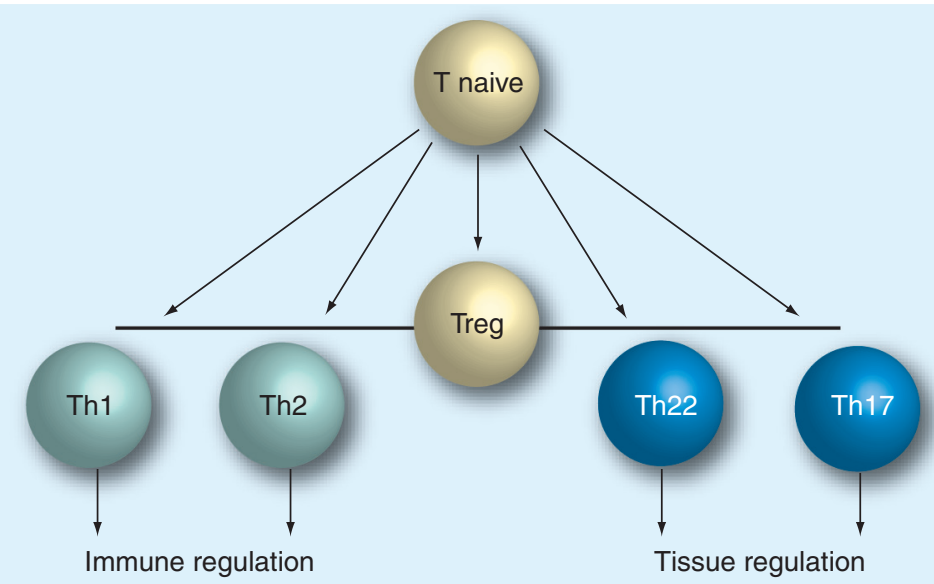

Figure 1. Grouping of T-cell subsets into immune regulators and those subsets that regulate tissue cells. Regulatory cells play an important role prior to effector functions. All subsets appear to develop from naive T cells that differentiate into helper subsets.

Treg: Regulatory T cell. the site of antigen contact. An array of other genes are also induced in the target tissue cells, which altogether results in tissue inflammation that is underlying the pathology of many T-cell-driven diseases such as rheumatoid arthritis (reviewed in [20]). The discovery of Th17 cells not only emphasizes the important role of $\mathrm{T}$ cells in regulating immune responses in the tissues, but also increases the interest in IL-22, which is remarkable in the sense that its receptor is exclusively expressed by structural, nonimmune cells [21].

\section{Th22 cells}

In chronic diseases such as psoriasis, contact and allergic dermatitis, Th17 cells were more closely investigated and were directly isolated from the site of inflammation. Interestingly, cells expressing IL- 4 and IFN- $\gamma$ or IL-17 and IFN- $\gamma$ were regularly found in these tissues at the same time. Very few cells showed IL-4 and IL-17 coexpression. In this context, the term 'plasticity' was generated, as already established T-helper subsets such as Th1 or Th2 cells can be driven to express IL-17 at least transiently. Most interesting, however, was a T-cell subpopulation that exclusively expressed IL-22 and was deficient in IL-17, IL-4 and IFN- $\gamma$ expression [22]. Similarily to Th17 cells, the IL-22 cytokine is recognized by the IL-22R, which is exclusively found on tissue cells. These cells could be cloned (along with Th1, Th2, Th17 or Th17-IL-22 ${ }^{+}$cells), were remarkably stable over 10 weeks of culture and were also resistant to cultures attempting to differentiate in vitro towards other T-cell phenotypes. The expression profile of these clones was compared with other clones of know subset identity (Th1, Th2 and Th17) and revealed that these cells are as distinct from Th17 as Th1 cells are distinct from Th2 cells. The Th22 cells also showed a distinct transcription factor and functional profile. The differentiation pathway of Th22 cells is still unclear; however, simple T-cell receptor engagement is sufficient to induce IL-22. Furthermore, plasmacytoid dendritic cells and IL- 6 and TNF- $\alpha$ were suggested to promote Th22 commitment [23]. To explore the function these $\mathrm{T}$ cells, clone supernatants were added to keratinocytes, which revealed that $T h 22$ cells promoted epithelial growth and wound closure in an in vitro scratch assay. Th22 supernatants also induced keratinocyte genes, which enable innate immune defenses (Toll-like receptors and complement factors) and reinforce the barrier function of the epithelium (keratins and laminin [22]). The current data suggest that Th22 cells are not only defined by a unique expression profile, but also by a distinct functional profile that promotes wound 
healing and epithelial integrity. The $I L-22$ gene was identified as a lead cytokine for these cells. IL-22 is a homeostatic cytokine that can protect and also reinforce proinflammatory response [Eyerich S, Eyerich K, Cavani A, Schmidt-Weber C, Trends in IMmunology; Unpublished Data].

\section{Future perspective}

New T-cell subsets fall into the category of tissue regulation and are now recognized to be involved in local tissue inflammation (FIgure 1). The discovery of more T-cell subsets may complicate our view on specific immune responses and the fact that some of the mediators are overlapping raises the question of whether the definition of new or any subset makes any sense. Is it possible that every $T$ cell differentiates in its own individual, imprinted expression profile and that grouping them into subsets is not justified? To answer this question, we will need to analyze many more T-cell clones and cluster them in a hypothesis-free genome-wide approach. However, our knowledge of T-cell differentiation suggests that T-cell affects is affecting larger gene loci. For example, Th2 differentiation unlocks a locus control region, which mobilizes not only IL-4 but also IL-5 and IL-13. Immunotherapy approaches, therefore, benefit from the discovery of new T-cell subsets, as they reveal new target structures, which can be addressed to selectively interfere with consequences of inflammation such as remodeling.

\section{Acknowledgements}

Stefanie Eyerich is acknowledged for helpful discussion and critical review of this article.

\section{Financial \& competing interests disclosure}

The author has no relevant affiliations or financial involvement with any organization or entity with a financial interest in or financial conflict with the subject matter or materials discussed in the manuscript. This includes employment, consultancies, honoraria, stock ownership or options, expert testimony, grants or patents received or pending, or royalties. No writing assistance was utilized in the production of this manuscript.

\section{Bibliography}

1 Schmidt-Weber CB: Th17 and Treg cells innovate the Th1/Th2 concept and allergy research. Chem. Immunol. Allergy 94, 1-7 (2008).

2 Mosmann TR, Cherwinski H, Bond MW, Giedlin MA, Coffman RL: Two types of murine helper T cell clone. I. Definition according to profiles of lymphokine activities and secreted proteins. J. Immunol. 136(7), 2348-2357 (1986).

3 Sornasse T, Larenas PV, Davis KA, De Vries JE, Yssel H: Differentiation and stability of $\mathrm{T}$ helper 1 and 2 cells derived from naive human neonatal CD4 $4^{+} \mathrm{T}$ cells, analyzed at the single-cell level. J. Exp. Med. 184(2), 473-483 (1996).

4 Nakamura T, Kamogawa Y, Bottomly K, Flavell RA: Polarization of IL-4- and IFN- $\gamma$-producing $\mathrm{CD} 44^{+} \mathrm{T}$ cells following activation of naive $\mathrm{CD} 4^{+} \mathrm{T}$ cells. J. Immunol. 158(3), 1085-1094 (1997).

5 Tao X, Constant S, Jorritsma P, Bottomly K: Strength of TCR signal determines the costimulatory requirements for Th1 and $\mathrm{Th} 2 \mathrm{CD}^{+} \mathrm{T}$ cell differentiation. J. Immunol. 159(12), 5956-5963 (1997).

6 Abbas AK, Murphy KM, Sher A: Functional diversity of helper T lymphocytes. Nature 383, 787-793 (1996).

7 Kurata H, Lee H, O'Garra A, Arai N: Ectopic expression of activated STAT6 induces the expression of Th2-specific cytokines and transcription factors in developing Th1 cells. Immunity 11(6), 677-688 (1999).

8 Ouyang W, Ranganath S, Weindel K et al.: Inhibition of Th1 development mediated by GATA-3 through an IL-4-independent mechanism. Immunity 9(5), 745-755 (1998).

9 Usui T, Nishikomori R, Kitani A, Strober W: GATA-3 suppresses Th1 development by downregulation of STAT4 and not through effects on IL-12r $\beta 2$ chain or T-bet. Immunity 18(3), 415-428 (2003).

10 Hwang E, Szabo S, Schwartzberg P, Glimcher L: T helper cell fate specified by kinase-mediated interaction of t-bet with GATA-3. Science 307(5708), 430-433 (2005).

11 Schmidt-Weber C, Blaser K: Immunological mechanisms of specific allergen immunotherapy. Inflamm. Allergy Drug Targets 5(1), 15-21 (2006).

12 Schmidt-Weber CB, Blaser K: T-cell tolerance in allergic response. Allergy 57(9), 762-768 (2002).

13 Schmidt-Weber CB, Blaser K: Immunological mechanisms of specific allergen immunotherapy. Curr. Drug Targets Inflamm. Allergy (2004).

14 Schmidt-Weber CB, Blaser K: Immunological mechanisms in specific immunotherapy. Springer Semin. Immunopathol. 25(3-4), 377-390 (2004).

15 Schmidt-Weber CB, Blaser K: New insights into the mechanisms of allergen-specific immunotherapy. Curr. Opin. Allergy Clin. Immunol. 5(6), 525-530 (2005).
16 Feuerer M, Hill JA, Mathis D, Benoist C: Foxp $3^{+}$regulatory T cells: Differentiation, specification, subphenotypes. Nat. Immunol. 10(7), 689-695 (2009).

17 Schmidt-Weber CB, Akdis M, Akdis CA: Th17 cells in the big picture of immunology. J. Allergy Clin. Immunol. 120 (2), 247-254 (2007).

18 Ouaked N, Mantel PY, Bassin C et al.: Regulation of the foxp 3 gene by the Th1 cytokines: the role of IL-27-induced STAT1. J. Immunol. 182(2), 1041-1049 (2009).

19 Burgler S, Ouaked N, Bassin C et al.: Differentiation and functional analysis of human T(h)17 cells. J. Allergy Clin. Immunol. (2009).

20 Louten J, Boniface K, De Waal Malefyt R: Development and function of Th17 cells in health and disease. J. Allergy Clin. Immunol. 123(5), 1004-1011 (2009).

21 Wolk K, Kunz S, Witte E, Friedrich M, Asadullah K, Sabat R: IL-22 increases the innate immunity of tissues. Immunity 21(2), 241-254 (2004).

22 Eyerich S, Eyerich K, Pennino D et al:: Th22 cells represent a distinct human $\mathrm{T}$ cell subset involved in epidermal immunity and remodeling. J. Clin. Invest. 119(12), 3573-3585 (2009).

23 Duhen T, Geiger R, Jarrossay D, Lanzavecchia A, Sallusto F: Production of interleukin 22 but not interleukin 17 by a subset of human skin-homing memory $\mathrm{T}$ cells. Nat. Immunol. 10(8), 857-863 (2009). 